\title{
The effect of volatile anesthetics on cellular responses in the microcirculation of free tissue transfers
}

\author{
Jakub Szrama ${ }^{1}$, Krzysztof Kusza' ${ }^{2}$, Paweł Sobczyńskiं ${ }^{3}$, Zsolt Molnar ${ }^{1,4}$, Maria Siemionow ${ }^{5}$ \\ ${ }^{1}$ Department of Anesthesiology and Intensive Therapy, Poznan University of Medical Sciences, Poznan, Poland \\ ${ }^{2}$ Chair and Department of Anesthesiology and Intensive Therapy, Poznan University of Medical Sciences, Poznan, Poland \\ ${ }^{3}$ First Department of Anesthesiology and Intensive Therapy, Poznan University of Medical Sciences, Poznan, Poland \\ ${ }^{4}$ Semmelweis University, Department of Anesthesiology and Intensive Therapy, Budapest, Hungary \\ ${ }^{5}$ University of Illinois, United States
}

Adv Interv Cardiol 2022; 18, 4 (70): 459-464

DOI: https://doi.org/10.5114/aic.2021.110926

\begin{abstract}
A bstract
The purpose of this review was to summarize the anti-inflammatory and immunosuppressive properties of volatile anesthetics and present their potential impact on the outcomes of major surgical procedures as well as microsurgical cases of free tissue transfer. Inhaled anesthetics are commonly used as a component of general anesthesia in interventional procedures, reconstructive surgery, free tissue transfers and transplantation. Experimental and clinical studies have shown that volatile anesthetics such as halothane, sevoflurane, isoflurane or desflurane can affect the immune system of patients exposed to general anesthesia. In patients with no serious systemic diseases, this effect is transient and mostly clinically irrelevant. However, in patients subjected to the inflammatory response due to the active disease, cardiac or pulmonary failure or advanced age, the prognosis may improve or worsen following inhalation anesthesia depending on the type of systemic pathology. The available data from reported clinical trials, as well as the in vitro and in vivo experimental studies, have often reported conflicting statements regarding the impact of inhalation anesthetics on outcomes of surgical procedures. These differences may be due to the heterogeneity of the evaluated patients, the extent and duration of surgical procedures, and different experimental design and methodologies applied for assessment of the reported clinical and research studies. In this review, based on the available literature reports we have summarized the anti-inflammatory and immunosuppressive effects as well as cellular responses of inhalation anesthetics at the microcirculatory level and discussed their potential clinical implications for the outcomes of surgical procedures of free tissue transfers.
\end{abstract}

Key words: volatile anesthetics, immunomodulation, cellular responses, microcirculation, free tissue transfers.

\section{Introduction}

Several scientific reports confirm immunosuppressive effects of volatile anesthetics on various types of immune cells including neutrophils, macrophages, natural killer (NK) cells and lymphocytes. These responses differ and depend on the choice of volatile anesthetics for the particular surgical procedure as well as on the duration of exposure and the dose. Volatile anesthetics have been shown to exert beneficial immunomodulatory properties under inflammatory conditions due to ischemia-reperfusion, hypoxia-reoxidation or other acute inflammatory responses [1-4].

Clinical studies testing the effect of volatile anesthetics during major interventional and surgical procedures including lung, heart or liver surgery [5-7] confirmed a re- duced number of postoperative complications compared to the cases under standard general anesthesia. There is, however, a limited number of reports on the application of inhaled anesthetics in different microsurgical procedures including free tissue transfers and there is also a lack of uniform guidelines on the choice of anesthetics or informative conclusions. It is clear that due to the known anti-inflammatory and immunomodulatory effects, volatile anesthetics could modulate the responses to surgical trauma and affect the microcirculatory hemodynamics of surgically reconstructed tissues and organs.

This is specifically important when patients with major comorbidities such as a history of cardiac or pulmonary failure or patients with known or expected immune deficiencies are scheduled for interventional procedures

Corresponding author:

Prof. Maria Siemionow, University of Illinois, United States, phone: +1 216212 7012, e-mail: siemiom@uic.edu

Received: 2.03.2021, accepted: 28.10.2021. 
or major reconstructive surgical procedures. The most frequently seen patients are those who require surgical intervention for cancer treatment. These patients are often immunosuppressed, and anesthetics could theoretically modify the immune response in the perioperative period, hence affecting the oncological outcomes [8].

However, these positive effects of volatile anesthetics are very difficult to identify or evaluate in clinical practice and they are often neglected by the treating anesthesiologists and surgeons alike.

Therefore, based on the literature reports, the aim of this review was to summarize the results of experimental and clinical studies assessing immunomodulatory effects of volatile anesthetics on the immune response generated under different surgical and microsurgical procedures including free tissue transfers [9-11].

\section{Microcirculatory response to volatile anesthetics as a function of neutrophil activation}

Neutrophils play a pivotal role in innate immunity. They take part in phagocytosis, produce oxygen radicals, and destroy foreign entities. However, their overwhelming activity can also provoke hyperinflammation, which can cause tissue damage and organ dysfunction.

There are many experimental studies which prove that volatile anesthetics including halothane, isoflurane and sevoflurane inhibit the production of reactive oxygen species (ROS) by activated neutrophils and in consequence reduce the capability of neutrophils to destroy bacteria [12, 13].

The impaired release of ROS from neutrophils results in reduced adhesion of neutrophils to the vascular endothelium, which has a direct effect on the reduced activation of rolling, sticking and transmigrating leukocytes and in consequence amelioration of the ischemia-reperfusion injury [2-4, 14].

A study evaluating the effect of pre-treatment with isoflurane on endotoxin-induced acute lung injury (ALI) showed that isoflurane reduced neutrophil migration to the lung interstitium and alveolar space and reduced lung the edema formation and capillary protein leakage. Furthermore, the exposure to isoflurane was associated with a reduced level of two critical chemotactic chemokines, CXCL1 and CHCL 2/3 in the alveolar space [12].

$A$ rat model of liver transplantation revealed that exposure to sevoflurane attenuated neutrophil induced renal injury and decreased neutrophil infiltration, as well as proinflammatory cytokine levels [15]. Liver ischemia/ reperfusion injury occurs following liver resection, hemorrhagic shock and transplantation and affects postoperative liver function and cardiovascular complications. A rat liver model of ischemia-reperfusion injury by Liao et al. found that sevoflurane administration decreased interleukin (IL) 1 (IL-1), IL-6 and tumor necrosis factor- $\alpha$
(TNF- $\alpha$ ) levels; it further suppressed the nuclear factor- $\kappa B$ $(\mathrm{NF}-\kappa \mathrm{B})$ signaling pathway activation. This reduced the ischemia/reperfusion injury to the liver [16]. Different experimental models confirm that primary inflammatory damage often leads to organ dysfunction.

Numerous studies have also demonstrated the role of neutrophils in the ischemia and reperfusion injury in the myocardium [6]. It was reported that sevoflurane and isoflurane impair post-ischemic adhesion of neutrophils in the intact coronary system of the isolated reperfused guinea pig hearts [6]. These properties of volatile anesthetics may be a part of the phenomenon called anesthetic preconditioning of the ischemic myocardium and may have beneficial effects on the myocardium and cardiac function during anesthesia [17].

Since free tissue transfers represent microsurgical procedures where ischemia-reperfusion is always an unavoidable part of the surgery, data reporting a protective effect of isoflurane and sevoflurane on reduction of ROS production by neutrophils may play an important role in the outcomes of free flap procedures $[6,11]$.

A study comparing the effects of sevoflurane and desflurane on the serum cytokine levels and urine chemokine levels in patients undergoing living-donor kidney transplant found that both volatile anesthetics attenuated the proinflammatory response in those patients. The authors observed no difference in the transplanted kidney function and similar preoperative and postoperative levels of proinflammatory cytokines, including TNF- $\alpha$, IL-2 and chemokines 9 and 10, suggesting protective effects of volatile anesthetics on ischemia-reperfusion injury in living-donor kidney transplant recipients [18]. We have also reported different microcirculatory effects during procedures of free flap transplantation under isoflurane anesthesia [19]. Specifically, Kusza et al., applying an intravital microscopy model of the cremaster muscle flap, assessed hemodynamic changes in the peripheral microcirculation of the skeletal muscle under halothane and isoflurane general anesthesia [2]. It was found that halothane anesthesia significantly altered leukocyte-endothelial activation, resulting in a reduced number of rolling and sticking leukocytes to the postcapillary venules and in consequence leading to improved capillary perfusion. The possible mechanism of improved microcirculatory hemodynamics could be via reduced levels of ROS leading to reduced endothelial edema and micro-thrombi formation and changes in the arteriolar response leading to dilatation of the resistance arterioles and precapillary sphincters [2].

Moreover, in the follow-up study, Kusza et al. assessed the microcirculatory response to halothane and isoflurane anesthesia using a rat model of induced cardiac arrest [3]. Following cardiac arrest, exposure of experimental animals to the 1 minimum alveolar concentration (MAC) of the halothane anesthesia ameliorated the harmful effects of cardiac arrest on the peripheral 
microcirculation, as confirmed by a significant decrease of the transmigrating leukocytes which are primarily responsible for the interstitial edema. Thus, reduced edema improved capillary perfusion [3]. The intravital cremaster muscle model was also tested by Mielniczuk et al. under experimentally induced hemorrhagic shock conditions and confirmed again the protective, anti-inflammatory effect of sevoflurane on the hemodynamics of the microsurgical model of free tissue transfer [4]. Effects of volatile anesthetics on the microcirculation are presented in Figure 1.

The protective effect of volatile anesthetics on the microcirculation may be of great clinical importance in the cases of reconstructive microsurgery. Sevoflurane could have beneficial effects on the microcirculation as compared to propofol by decreasing the extravasation of fluid into the interstitium $[9,20]$. Sevoflurane administration may protect the endothelium against ischemia-reperfusion injury, thereby improving tissue perfusion and oxygenation. One of the suggested mechanisms is the attenuation of leucocyte activation, which is responsible for tissue injury after ischemia-reperfusion [21]. This phenomenon may also appear in free flap microsurgical procedures.

Volatile anesthetics may influence the microcirculation by affecting angiogenesis and extracellular matrix synthesis. Anesthesia with volatile anesthetics stimulated proliferation of endothelial progenitor cells. However, another study showed a depleting effect of volatile anesthetics on hypoxia-inducible factor-1 activity, with a further inhibitory effect on vascular endothelial growth factor (VEGF) and angiogenesis. An in vitro study on renal proximal tubule cells showed increased synthesis of extracellular matrix after exposure to volatile anesthetics due to increase of transforming growth factor- $\beta 1$ (TGF- $\beta 1$ ) [22].

An interesting study comparing the effects of different types of anesthesia on the microcirculation in a rat cremaster muscle flap model showed that rolling and adhesion of leucocytes to the endothelium was significantly reduced under general anesthesia with volatile anesthetics compared to the spinal anesthesia models. Furthermore, addition of epidural anesthesia to the general volatile anesthesia resulted in increased perfusion of the microcapillaries [23].

The above summarized reports on the protective effects of the volatile anesthetics at the microcirculatory level of muscle flaps clearly indicate the importance of anesthesia choice during microsurgical procedures of free tissue transfers $[2-4,9,10]$.

\section{Immunomodulatory effect of volatile anesthetics on the macrophage responses}

Macrophages are phagocytic cells of innate immunity, similar to neutrophils. They are often the first line of defensive mechanisms, sending recruitment signals to various other effector cells [24].
Kalimeris et al. studied sevoflurane and desflurane and found a decrease in macrophage concentration in bronchoalveolar lavage in mechanically ventilated pigs [25]. A study evaluating the effect of sevoflurane on the inflammatory response of the lungs after cardiopulmonary bypass revealed that sevoflurane decreased leukocyte count, IL-6, IL-8 and IL-10 levels; this attenuated the systemic inflammatory response induced by cardiopulmonary bypass [26]. Thus, the beneficial systemic effects of volatile anesthetics on reduction of inflammatory responses in organs such as the lung and heart will further lead to improved outcomes in free tissue transfers, specifically in the high risk elderly and compromised patients [10].

\section{Immunosuppressive effect of volatile anesthetics on the function of natural killer cells}

Cell-mediated immunity (including cytotoxic T-lymphocytes, natural killer (NK) cells, natural killer T (NKT) cells, macrophages and dendritic cells) plays a critical role in eliminating tumor cells. Low preoperative NK cell activity was associated with the development of tumor recurrence and distant metastasis in colorectal cancers [27].

Volatile anesthetics were proved to have an immunosuppressive effect by reducing the number of NK cells and by inhibiting the stimulation of NK cells by interferon (IFN). An increased concentration of halothane and enflurane is related to decreased NK cell activity [28].

Both isoflurane and sevoflurane are responsible for triggering T-lymphocyte and NK cell apoptosis, leading to immunosuppression and cancer progression. Both in vitro and in vivo studies showed that halothane and

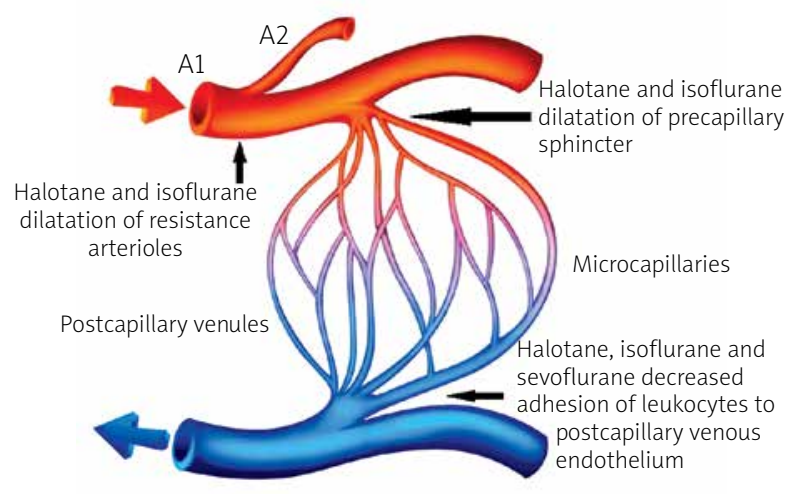

Figure 1. The effect of volatile anesthetics on the microcirculation. Volatile anesthetics are responsible for dilatation of the resistance arterioles and the precapillary sphincters. They also decrease adhesion of leukocytes to the postcapillary venules' endothelium, resulting in prevention of endothelial edema and improved microcirculatory perfusion (Figure 1 image was purchased on the Internet VectorStock site) 
isoflurane suppress the INF-induced stimulation of NK cell activity, resulting in an increased rate of metastasis [29]. Volatile anesthetics are also responsible for decreased production of cytokines by the NK cells stimulated by the tumor cells [27].

\section{Immunosuppressive effect of volatile anesthetics on lymphocyte function}

Many studies have confirmed immunosuppressive effect of volatile anesthetics on the proliferation and release of cytokines by lymphocytes [12]. This may further lead to induction of apoptosis, which was confirmed in studies assessing the dose- and time-dependent effect of isoflurane and sevoflurane on apoptosis of peripheral blood lymphocytes [30]. The number of lymphocytes with caspase-3 like activity increased after exposure to both sevoflurane and isoflurane. The data from this study imply the possibility that lymphocytic apoptosis induced by

Table I. The effect of volatile anesthetics on the immune system

\begin{tabular}{ll} 
Immune cell type & \multicolumn{1}{c}{ Effect } \\
\hline Neutrophils & $\begin{array}{l}\text { Decreased production of ROS } \\
\text { Reduced adhesion to the endothelium } \\
\text { Decreased activity }\end{array}$ \\
\hline Macrophages & $\begin{array}{l}\text { Reduced activation, migration } \\
\text { Reduced cytokine synthesis } \\
\text { Reduced SIRS response }\end{array}$ \\
\hline NK-cell & $\begin{array}{l}\text { Impaired cytotoxic activity } \\
\text { Decreased activation by INF- } \gamma\end{array}$ \\
\hline Lymphocytes & $\begin{array}{l}\text { Decreased activation } \\
\text { Reduced secretion of cytokines } \\
\text { Reduced production of antibodies } \\
\text { Induction of apoptosis }\end{array}$
\end{tabular}

The immunomodulatory effect of the volatile anesthetics on the cells of the immunological system. The details are presented in the respective sections of the manuscript. ROS - reactive oxygen species, SIRS - systemic inflammatory response syndrome, NK-cells - natural killer cells, INF- $\gamma$ - interferon $\gamma$. volatile anesthetics may cause perioperative lymphocytopenia [30].

Based on these results, Loop et al. reported that sevoflurane and isoflurane induce a cellular apoptosis pathway by influencing the release of cytochrome $c$ from mitochondria into the cytoplasm in human T lymphocytes in a concentration-dependent fashion [12]. The loss of cytochrome c from the electron transfer chain, induced by volatile anesthetics, is believed to destabilize the mitochondrial membrane potential and significantly impairs the lymphocytic synthesis of adenosine triphosphate (ATP), leading to activation of caspases, and cell death via induction of apoptosis.

Volatile anesthetics have the potential to affect the ratio of Th1/Th2 lymphocytes, which might have an effect on cancer prognosis. A study by Lee et al. showed that patients with hepatocellular carcinoma treated with intraarterial chemoembolization had a more favorable outcome indicated by increased lymphocyte Th1/Th2 and IFN- $\gamma / \mathrm{IL}-10$ ratios [31]. After isoflurane anesthesia the Th1/Th2 ratio decreased significantly, but it remained unchanged after the use of propofol anesthesia [32]. A study on breast cancer patients revealed that desflurane anesthesia can preserve the favorable Th1/Th2 ratio, as well as their cytokine ratio [33]. An interesting study performed by Zhou et al. on 61 patients with hepatocellular carcinoma undergoing hepatectomy under general anesthesia or combined epidural and general anesthesia showed that patients receiving neuraxial blockade had a more favorable Th1/Th2 lymphocyte ratio with respect to promoting anti-tumor Th polarization [34].

A study comparing general and combined epidural with general anesthesia for the surgical treatment of gall bladder cancer found that addition of regional anesthesia resulted in improved immune cell function as compared to general anesthesia alone. The survival rate of $\mathrm{CD} 3+, \mathrm{CD} 4+$, and CD4+/CD8+ lymphocytes was signifi-

Table II. Clinical implication of the immunomodulatory effects of volatile anesthetics

\begin{tabular}{|c|c|}
\hline Organ affected & Effect \\
\hline Heart & Reduced ischemia-reperfusion injury \\
\hline Lung & $\begin{array}{l}\text { Reduced lung edema, reduced capillary leakage in ALI } \\
\text { Reduced intraalveolar recruitment of macrophages in influenza } \\
\text { Decreased proinflammatory cytokines in bronchoalveolar lavage } \\
\text { Decreased macrophage concentration in bronchoalveolar lavage during mechanical ventilation } \\
\text { Reduced inflammatory mediators in lungs after CABG }\end{array}$ \\
\hline Liver & Decreased ischemia/reperfusion injury in rat liver \\
\hline Kidney & $\begin{array}{l}\text { Reduced kidney injury after liver transplantation } \\
\text { Protective effect in ischemia-reperfusion injury in living donor kidney transplant recipients }\end{array}$ \\
\hline $\begin{array}{l}\text { Blood vessels - } \\
\text { microcirculation }\end{array}$ & $\begin{array}{l}\text { Increased perfusion of microcirculation - dilatation of resistance arterioles and precapillary sphincters } \\
\text { Decreased swelling of the postcapillary venule endothelium }\end{array}$ \\
\hline Free flap surgery & Increased microcirculatory perfusion, decreased tissue edema, reduced ischemia-reperfusion injury \\
\hline Neoplasm & Increased cancer progression, increased rate of metastasis \\
\hline
\end{tabular}

Clinical implication of the immunomodulatory effects of volatile anesthetics in various organs and tissues including free flaps. For details see the respective sections of the manuscript. ALI - acute lung injury, CABG - coronary artery bypass graft. 
cantly higher in the combined epidural-general anesthesia group, which could imply that this type of anesthesia could improve patients' postoperative immunological condition and furthermore their long-term prognosis [35]. Although further studies in the area of volatile anesthetics affecting Th lymphocyte immunoreactions in cancer patients are needed, there is strong evidence suggesting that the type of anesthesia chosen may affect the overall cancer outcomes.

Several studies have addressed the effect of different types of anesthesia and different anesthetics on outcomes in cancer patients, as evaluated by recurrence or metastatic ratio. In some studies, volatile anesthetics were implicated in immunosuppression and direct stimulation of cancer cell survival and proliferation [36].

An interesting study evaluating the effect of serum from patients administered distinct anesthetic techniques on apoptosis in breast cancer cells in vitro revealed that serum from patients given sevoflurane anesthesia for primary breast cancer surgery reduces apoptosis in breast cancer cells to a greater extent than serum from patients given propofol-paravertebral anesthesia [37].

There are also some contradictory studies showing anti-cancer effects of volatile anesthetics. In the work by Ding et al., the authors found that sevoflurane significantly inhibited proliferation and migration of cervical cancer cells regardless of cellular origin and genetic background. The authors believe that these findings provide preclinical evidence for potential mechanisms by which sevoflurane may negatively affect cervical cancer growth and metastasis [38].

The effects of volatile anesthetics on the immune system and immunomodulatory effect on organs and microsurgical cases of free flaps are summarized in Tables I and II.

\section{Summary}

Volatile anesthetics can affect different aspects of the immunological system by both the direct effect on the immune cells and by indirect modulation of the perioperative stress response. The in vitro effects of anesthetics on the immune cells depend on the time of exposure, concentration of anesthetics and experimental models and technique used for the cell culture. In contrast, it is difficult to assess the immunomodulatory effects of volatile anesthetics in vivo in patients who present with comorbidities such as diabetes, cardiac or pulmonary failure and are submitted to complex surgical procedures due to cancer or severe multi-organ trauma.

Thus, knowledge of the effects of volatile anesthetics on the immune system of patients undergoing different interventional and surgical procedures including free tissue transfers is essential to avoid potential complications, specifically in the high-risk patients presenting with an already compromised immune system.

\section{Conflict of interest}

The authors declare no conflict of interest.

\section{References}

1. Kitakaze M. How to mediate cardioprotection in ischemic hearts: accumulated evidence of basic research should translate to clinical medicine. Cardiovasc Drugs Ther 2010; 24: 217-23.

2. Kusza K, Siemionow M, Nalbantoglu U, et al. Microcirculatory response to halothane and isoflurane anesthesia. Ann Plast Surg 1999; 43: 57-66.

3. Kusza K, Blaszyk M, Siemionow M, et al. Effects of halothane and isoflurane on leukocytes behaviour in peripheral microcirculation after experimental cardiac arrest. Eur J Anaesthesiol 2000; 1: 47-8.

4. Mielniczuk M, Krokowicz $九$, Grykien C, et al. Effects of volatile anesthetics on microcirculation during hypovolemia: a comparison of isoflurane and sevoflurane. Eur J Anaesthesiol 2008; 25 Suppl. 44: 62.

5. De Conno E, Steurer MP, Wittlinger M, et al. Anesthetic-induced improvement of the inflammatory response to one-lung ventilation. Anesthesiology 2009; 110: 1316-26.

6. De Hert SG, Turani F, Mathur S, et al. Cardioprotection with volatile anesthetics: mechanisms and clinical implications. Anesth Analg 2005; 100: 1584-93.

7. Beck-Schimmer B, Breitenstein S, Urech S et al. A randomized controlled trail on pharmacological preconditioning in liver surgery using a volatile anesthetic. Ann Surg 2008; 248: 909-18.

8. Jun IJ, Jo JY, Kim J, et al. Impact of anesthetic agents on overall and recurrence - free survival in patients undergoing esophageal cancer surgery: a retrospective observational study. Sci Rep 2017; 7: 14020.

9. Hagau N, Longrois D. Anesthesia for free vascularized tissue transfer. Microsurgery 2009; 29: 161-7.

10. Sierakowski A, Nawar A, Parker $M$, et al. Free flap surgery in the elderly: experience with 110 cases aged $\geq 70$ years. J Plast Reconstr Aesthet Surg 2017; 70: 189-95.

11. Adanali G, Ozer K, Siemionow M. Early and late effects of ischemic preconditioning on microcirculation of skeletal muscle flaps. Plast Reconstr Surg 2002; 109: 1344-51.

12. Kurosawa S, Kato M. Anesthetics, immune cells, and immune responses. J Anesth 2008; 22: 263-77.

13. Guochang H, Salem MR, Crystal GJ. Isoflurane prevents platelets from enhancing neutrophil-induced coronary endothelial dysfunction. Anesth Analg 2005; 101: 1261-8.

14. Hu G, Vinten-Johansen J, Salem MR, et al. Isoflurane inhibits neutrophil-endothelium interactions in the coronary circulation: lack of role for adenosine triphosphate-sensitive potassium channels. Anesth Analg 2002; 94: 849-56.

15. Kong HY, Zhu SM, Wang LQ, et al. Sevoflurane protects against acute kidney injury in a small-size liver transplantation model. Am J Nephrol 2010; 32: 347-55.

16. Liao X, Zhou S, Zong J, et al. Sevoflurane exerts protective effects on liver ischemia/reperfusion injury by regulating NFKB3 expression via miR-9-5p. Exp Ther Med 2019; 17: 2632-40.

17. Kevin LG, Novalija E, Stowe DF. Reactive oxygen species as mediators of cardiac injury and protection: the relevance to anesthesia practice. Anesth Analg 2005; 101: 1275-87.

18. Karadeniz M, Ciftci HS, Tefik T, et al. Effects of different volatile anesthetics on cytokine and chemokine production after ischemia-reperfusion injury in patients undergoing living-do- 
nor kidney transplant. Exp Clin Transplantat 2019; 17 (Suppl 1): 68-74.

19. Ozer K, Zielinski M, Siemionow M. New composite tissue allograft transplantation model in mouse with intravital microscopic evaluation of microcirculation. J Reconstr Microsurg 2003; 19: 323-30.

20. Bruegger A, Bauer U, Finsterer P, et al. Microvascular changes during anesthesia: sevoflurane compared with propofol. Acta Anaesthesiol Scand 2002; 46: 481-7.

21. Lucchinetti E, Ambrosio S, Aguirre J, et al. Sevoflurane inhalation at sedative concentrations provides endothelial protection against ischemia-reperfusion injury in humans. Anesthesiology 2007; 106: 262-8.

22. Bentov I, Reed MJ. Anesthesia, microcirculation, and wound repair in aging. Anesthesiology 2014; 120: 760-72.

23. Bozkurt M, Kulahci Y, Zor F, et al. Comparison of the effects of inhalation, epidural, spinal and combined anesthesia techniques on rat cremaster muscle flap microcirculation. Microsurgery 2010; 30: 55-60.

24. Stollings LM, Jia L, Tang P, et al. Immune modulation by volatile anesthetics. Anesthesiology 2016; 125: 399-411.

25. Kalimeris K, Christodoulaki K, Karakitsos P, et al. Influence of propofol and volatile anaesthetics on the inflammatory response in the ventilated lung. Acta Anaesthesiol Scand 2011; 55: 740-8.

26. Cho EJ, Yoon JH, Hong SJ, et al. The effects of sevoflurane on systemic and pulmonary inflammatory responses after cardiopulmonary bypass. J Cardiothorac Vasc Anesth 2009; 23: 639-45.

27. Jiao B, Yang C, Huang N, et al. Relationship between volatile anesthetics and tumor progression: unveiling the Mystery. Curr Med Sci 2018; 38: 962-7.

28. Dang Y, Shi X, Xu W, et al. The effect of anesthesia on the immune system in colorectal cancer patients. Can J Gastroenterol Hepatol 2018; 2018: 7940603.

29. Melamed R, Bar-Yosef S, Shakhar G, et al. Suppression of natural killer cell activity and promotion of tumor metastasis by ketamine, thiopental, and halothane, but not by propofol: mediating mechanisms and prophylactic measures. Anesth Analg 2003; 97: 1331-9.

30. Matsuoka H, Kurosawa S, Horinouchi T, et al. Inhalation anesthetics induce apoptosis in normal peripheral lymphocytes in vitro. Anesthesiology 2001; 95: 1467-72.

31. Lee $H$, Jang JW, Lee SW, et al. Inflammatory cytokines and change of Th1/th2 balance as prognostic indicators for hepatocellular carcinoma in patients treated with transarterial chemoembolisation. Sci Rep 2019; 9: 3260.

32. Inada T, Yamanouchi $Y$, Jomura S, et al. Effect of propofol and isoflurane anaesthesia on the immune response to surgery. Anaesthesia 2004; 59: 954-9.

33. Woo JH, Baik HJ, Kim CH, et al. Effect of propofol and desflurane on immune cell populations in breast cancer patients: a randomized trial. J Korean Med Sci 2015; 30: 1503-8.

34. Zhou D, Gu FM, Gao Q, et al. Effects of anesthetic methods on preserving anti-tumor $\mathrm{T}$ helper polarization following hepatectomy. World J Gastroeneterol 2012; 18: 3089-9.

35. Zhu J, Zhang XR, Yang H. Effects of combined epidural and general anesthesia on intraoperative hemodynamic responses, postoperative cellular immunity, and prognosis in patients with gallbladder cancer. A randomized controlled trial. Medicine 2017; 96: e6137.
36. Cata PJ, Owusu-Agyemang P, Kapoor R, et al. Impact of anesthetics, analgesics and perioperative blood transfusion in pediatric cancer patients: a comprehensive review of the literature. Anesth Analg 2019; 129: 1653-65.

37. Jaura Al, Flood G, Gallagher HC, et al. Differential effects of serum from patients administered distinct anesthetic techniques on apoptosis in breast cancer cells in vitro: a pilot study. BJA 2014; 113 (Suppl 1): i63-7.

38. Ding J, Zhang L, Zeng S, et al. Clinically relevant concetration of sevolflurane suppresses cervical cancer growth and migration through targeting multiple oncogenic pathways. Biochem Biophys Res Commun 2019; 514: 1179-84. 